\title{
Catheter Ablation for Fascicular Ventricular Tachycardia: A Systematic Review
}

\author{
Antonio Creta ${ }^{1,2}$, Anthony Chow ${ }^{1}$, Simon Sporton $^{1}$, Malcolm Finlay $^{1}$, Nikolaos \\ Papageorgiou $^{1}$, Shohreh Honarbakhsh ${ }^{1}$, Gurpreet Dhillon ${ }^{1}$, Adam Graham ${ }^{1}$, Kiran HK Patel ${ }^{3}$, \\ Mehul Dhinoja ${ }^{1}$, Mark J. Earley ${ }^{1}$, Ross J. Hunter ${ }^{1}$, Martin Lowe ${ }^{1}$, Edward Rowland ${ }^{1}$, Oliver \\ R. Segal ${ }^{1}$, Vito Calabrese ${ }^{2}$, Danilo Ricciardi ${ }^{2}$, Pier D. Lambiase ${ }^{1}$, Richard J. Schilling ${ }^{1}$, Rui \\ Providência $^{1}$
}

1. Barts Heart Centre, St. Bartholomew's Hospital, London, United Kingdom

2. Campus Bio-Medico University of Rome, Rome, Italy

3. Northwick Park Hospital, London, United Kingdom

Running title: Fascicular tachycardia and Ablation

Manuscript word count: 2618 words

\section{Corresponding author:}

Antonio Creta

Campus Bio-Medico University of Rome \& Barts Heart Centre

St. Bartholomew's Hospital

West Smithfield

London EC1A 7BE

Tel: +442037658646

Email: creta.antonio@gmail.com

All authors take responsibility for all aspects of the reliability and freedom from bias of the data presented and their discussed interpretation. 


\begin{abstract}
Introduction: Catheter ablation has been evaluated as treatment for fascicular ventricular tachycardia (FVT) in several single-centre cohort studies, with variable results regarding efficacy and outcomes.
\end{abstract}

Methods: A systematic search was performed on PubMed, EMBASE and Cochrane database (from inception to November 2017) that included studies on FVT catheter ablation.

Results: Thirty-eight observational non-controlled case series comprising 953 patients with FVT undergoing catheter ablation were identified. Three studies were prospective and only 5 were multi-centre. Eight-hundred and eighty-four patients (94.2\%) had left posterior FVT, 25 (3.4\%) left anterior FVT and 30 (2.4\%) other forms. In 331 patients (41\%), ablation was performed in sinus rhythm (SR). The mean follow-up period was 41.4 \pm 10.7 months. Relapse of FVT occurred in 100 patients $(10.7 \%)$. Among the 79 patients $(8.3 \%)$ requiring a further procedure after the index ablation, $19(2 \%)$ had further FVT relapses. Studies in which ablation was performed in FVT had similar success rate after multiple procedures compared to ablation in SR only $\left(95.1 \%, \mathrm{CI}_{95 \%} 92.2-97 \%, \mathrm{I} 2=0 \%\right.$ versus $94.8 \%, \quad \mathrm{CI}_{95 \%} 87.6-97.9 \%, \quad \mathrm{I}^{2}=0 \%$, respectively). Success rate was numerically lower in paediatric-only series compared to nonpaediatric cases $\left(90.0 \%, \mathrm{CI}_{95} \% 82.1-94.6 \%, \mathrm{I}^{2}=0 \%\right.$ versus $94.3 \%, \mathrm{CI}_{95} \% 92.2-95.9 \%, \mathrm{I}^{2}=0 \%$, respectively).

Conclusion: Data derived from observational non-controlled case series, with lowmethodological quality, suggest that catheter ablation is a safe and effective treatment for FVT, with a 93.5\% success rate after multiple procedures. Ablation during FVT represents the firstline and most commonly used approach; however, a strategy of mapping and ablation during SR displayed comparable procedural results to actively mapping patients in FVT and should therefore be considered in selected cases where FVT is not inducible. 
Key words: fascicular ventricular tachycardia; verapamil-sensitive tachycardia; Belhassen's tachycardia; idiopathic left ventricular tachycardia; catheter ablation. 


\section{Introduction}

Fascicular ventricular tachycardia (FVT) was first described by Zipes et al in 1979 [1]. Belhassen et al demonstrated in 1981 that FVT can be terminated by verapamil [2]. FVT represents $10-15 \%$ of idiopathic ventricular tachycardia and is the most common form of idiopathic left ventricular (LV) tachycardia [3]. It has a male preponderance $(60-80 \%$ of reported cases occur in men) and usually presents in young adults (15-40 years) with structurally normal hearts [4-6]. Its prevalence is higher in Asian populations [7]. Three subtypes have been described: (1) left posterior FVT ( $90 \%$ of cases), with a right-bundle brunch block (RBBB) pattern and left axis deviation (Figure 1); (2) left anterior FVT (5-10\% of cases), with RBBB and right axis deviation; (3) upper septal FVT (<1\% of cases), with usually narrow QRS and normal frontal axis.

FVT is a re-entry tachycardia involving abnormal Purkinje tissue with decremental properties, left ventricular myocardium and the left anterior or posterior fascicles [8], though the entire components of the reentry circuit remain incompletely defined and understood [9].

In this report, we aim systematically to review the available data in the literature and assess the efficacy and rates of periprocedural complications following catheter ablation for FVT.

\section{Methods}

\section{Study Selection}

A systematic electronic search was performed on PubMed, EMBASE and Cochrane database (from inception to November 2017) with no language limitations, using the following search string: "fascicular ventricular tachycardia" OR "idiopathic left ventricular tachycardia" OR “verapamil-sensitive tachycardia" AND (“ablation" OR "catheter ablation”).

The population, intervention, comparison and outcome (PICO) approach was used [10]. The population of interest was patients with FVT, and the intervention was catheter ablation of 
FVT. In the absence of a control group, a non-controlled observational analysis was performed. The primary outcome measure was freedom of FVT at the end of follow-up. The other outcome considered was mortality. Procedural complications were assessed on a study-by-study basis. Eligibility criteria required studies to provide patient demographics and the FVT morphology. Observational non-controlled case series required a minimum of 6 patients to be considered eligible.

Review articles, editorials and case reports were not considered eligible for the purpose of this review.

Reference lists of all accessed full-text articles were further searched for sources of potentially relevant information.

Two independent reviewers (AC and RP) screened all abstracts and titles to identify potentially eligible studies, and the full text of was subsequently interrogated. Agreement of the two reviewers was required for studies to be considered eligible for analysis. Study quality was formally evaluated using the National Heart, Lung, and Blood Institute Quality Assessment Tool for Case Series Studies [11] by two reviewers (AC, RP). An agreement between the two reviewers was mandatory for the final classification of studies. A third author (AWC) intervened to resolve disputes whenever the two reviewers were in disagreement regarding the inclusion or classification of a study.

Data extraction and presentation for the preparation of this manuscript followed the recommendations of the PRISMA group [12]. Where available the following data were extracted from the selected studies: study design, study population characteristics (age and sex), FVT morphology and cycle length, follow-up duration, ablation procedure, definition of relapse, post-procedural monitoring, use of anti-arrhythmic agents. Patient-level data were obtained whenever these were available in the manuscripts, or provided by authors after contact. 


\section{Statistical analysis}

Statistical heterogeneity on each outcome of interest was quantified using the $\mathrm{I}^{2}$ statistic, which describes the percentage of total variation across studies due to heterogeneity rather than chance. Values of $<25 \%, 25 \%$ to $50 \%$, and $>50 \%$ are by convention classified as low, moderate, and high degrees of heterogeneity, respectively.

If no heterogeneity was found $\left(\mathrm{I}^{2} \leq 40 \%\right)$ OR were calculated using a fixed-effect model. If heterogeneity between studies was observed $\left(\mathrm{I}^{2}>40 \%\right)$, ORs were calculated using randomeffects model. Comprehensive Meta-Analysis software (Version 2) was used for the analyses. Overall incidences and 95\% confidence interval were estimated.

Sub-group analyses were performed for mapping strategy (mapping sinus rhythm vs. mapping in VT), use or not of mapping systems, and paediatric vs. non-paediatric cohorts.

\section{Results}

Study selection and patient characteristics

A total of 38 studies meeting the inclusion criteria were identified. The selection process is illustrated in Figure 2 (PRISMA) and a total population of 953 patients with FVT undergoing catheter ablation were included. Twenty-eight patients from 7 studies [7] [13-18] did not receive catheter ablation and therefore were not considered for the present analysis. Noninducibility of FVT was the most common reason for deferring ablation. The mean age of the patients was $29.5 \pm 12.4$ years; $82 \%$ were male. Eight-hundred and eighty-four patients $(94.2 \%)$ had left posterior FVT, 25 (3.4\%) left anterior FVT and 20 (2.1\%) had upper septal FVT. The sub-type of FVT was not specified in the remaining 24 patients. The mean cycle length was $364 \pm 66$ ms. Eighty-nine patients $(9.3 \%)$ had a previous failed or attempted FVT ablation before the index procedure considered in the present study. Data regarding antiarrhythmic treatment 
before catheter ablation were available for 18 studies (equating to 399 patients) and verapamil was used in the majority. Among the 363 patients with previous long-term antiarrhythmic drug treatment, 305 (84\%) had symptomatic FVT relapses despite medical therapy.

There was a perfect agreement between investigators on the inclusion of the selected studies. Baseline data and the design of selected trials are summarized in Table 1.

The 38 studies used for the analysis were all case series. Three studies were prospective [7] $[19,20]$. All the studies were observational, with no control group and all but 5 [15] [17] [2123] were single-centre. According to the National Heart, Lung, and Blood Institute Quality Assessment Tool for Case Series Studies [11] a maximum of 9 criteria apply for case series as shown in S-Table 1. Twenty-one studies fulfilled 8 criteria [7, 8] [13] [17-20] [24-37], 12 studies fulfilled 7 criteria [15] [21] [23] [38-46] and 5 studies fulfilled 6 criteria [14] [16] [24] [47, 48]. Both authors (AC and RP) were in agreement regarding study classification.

\section{Procedural Data}

During the index procedure, FVT was inducible or incessant in 871 patients $(91 \%)$. Of the 38 studies included in our analysis, only 11 studies reported on the duration of the procedure as well as the mean fluoroscopy time (Table 2). A tridimensional (3-D) mapping system was used in 416 cases (Biosense Webster CARTO system in 224 cases and Abbott-St. Jude Ensite NavX or Velocity in 149 cases, unknown in the remaining), while no 3-D mapping system was used in 531 patients. No data were reported regarding use of mapping system in one study (6 patients) [48]. Irrigated or non-irrigated 3.5 or $4 \mathrm{~mm}$ tip catheters were used for mapping and ablation (Table 2). A combination of radiofrequency and cryo-ablation was used in 3 cases [15]. Thirty-three studies (equating to 816 patients) described whether the catheter ablation was performed during sinus rhythm (SR) or during tachycardia. In 331 patients (41\%), the ablation was performed during SR. The targets of ablation varied among the studies. Fascicular 
block was induced in 196 patients (20.6\%). For most of the studies, termination and/or noninducibility of FVT was the acute procedural outcome. Detailed procedural data are presented in Table 2 and S-Table 2.

\section{Efficacy of Catheter Ablation}

The mean follow-up period was $41.4 \pm 10.7$ months. During the follow-up period, a total of 20 patients were lost in one study. Among those with follow-up, relapse of FVT occurred in 100 of 933 patients $(10.7 \%)$. Among the 79 patients $(8.3 \%)$ requiring a further procedure after the index ablation, 19 (2.0\%) had further FVT relapses. These data are summarized in Table 3.

In 17 studies freedom from FVT relapse was 100\% after the index ablation procedure [8] [13] [17] [20] [22] [25-31] [33, 34] [40] [43-45] Suzuky et al reported the highest rate of relapse $(50 \%)$ after catheter ablation [48].

Overall, the pooling of our data (Figure $3 \mathrm{a}-3 \mathrm{~b}$ ) shows that catheter ablation is effective to abolish FVT, with a success rate of $93.5 \%$ after multiple procedures. Interestingly, data regarding antiarrhythmic therapy was available for 18 studies included in the present analysis, and the overall long-term success rate of medical therapy was only $16 \%$.

\section{Sub-group Analyses}

Similar results were found between ablation during FVT only versus SR only, with a success rate after multiple procedures of $95.1 \%\left(\mathrm{CI}_{95 \%} 92.2-97 \%, \mathrm{P}<0.001, \mathrm{I}^{2}=0 \%\right)$ and $94.8 \%$ $\left(\mathrm{CI}_{95 \%}\right.$ 87.6-97.9\%, $\left.\mathrm{P}<0.001, \mathrm{I}^{2}=0 \%\right)$, respectively. In the 9 studies in which catheter ablation was performed in either FVT or SR, the success rate was $86.7 \%\left(\mathrm{CI}_{95 \%} 82.1-90.2 \%, \mathrm{P}<0.001\right.$, $\left.\mathrm{I}^{2}=0 \%\right)$ after the index procedure and $93.2 \%\left(\mathrm{CI}_{95 \%} 85.7-96.9 \%, \mathrm{P}<0.001, \mathrm{I}^{2}=38 \%\right)$ after multiple procedures. 
Use of 3-D mapping did improve numerically the success rate compared to fluoroscopy-only procedures, especially after the index procedure $\left(92 \%, \mathrm{CI}_{95} \% 87.8-94.9 \%, \mathrm{P}<0.001 \mathrm{I}^{2}=0 \%\right.$ versus $89.5 \%, \mathrm{CI}_{95 \%} 83.7-93.4 \%, \mathrm{P}<0.001, \mathrm{I}^{2}=0 \%$, respectively).

Success rate was numerically lower in paediatric-only series compared to non-paediatric cases (success rate after multiple procedures $90.0 \%, \mathrm{CI}_{95 \%} 82.1-94.6 \%, \mathrm{P}<0.001, \mathrm{I}^{2}=0 \%$ versus 94.3\%, CI95\%92.2-95.9\%, $\mathrm{P}<0.001, \mathrm{I}^{2}=0 \%$, respectively) (Table 4).

No significant differences were found between use of irrigated versus non-irrigated ablation catheter, with a success rate after the index procedure of $88.8 \%\left(\mathrm{CI}_{95} \% 69.9-96.5 \%, \mathrm{P}<0.001\right.$, $\left.\mathrm{I}^{2}=1 \%\right)$ and $87.7 \%\left(\mathrm{CI}_{95 \%} 84.4-90.4 \%, \mathrm{P}<0.001, \mathrm{I}^{2}=0 \%\right)$, respectively. No data were available regarding patients treated with irrigated catheters having repeated procedures, and therefore comparison of success rates after multiple procedures was not possible.

\section{Procedural complications}

Procedural complications occurred in 13 patients (1.4\%). Suzuky et al [48] reported that one patient (17\%) developed complete left bundle branch block (LBBB) and that another patient (17\%) developed complete heart block requiring permanent pacemaker implant. Collins et al [15] reported complications in 6 patients (7\%), including $2(2 \%)$ VF arrest, 3 (3\%) polymorphic VT and one (1\%) transient atrio-ventricular block; also, one patient developed LBBB which did not fit with the complication definition of the study and therefore has not been included in the present analysis. Topilski et al [18] and Shin et al [21] reported iatrogenic VF arrest requiring direct-current shock in one patient each. Guo et al [13] reported one femoral pseudoaneurysm, while Arya et al reported one haematoma at the femoral access site. In addition, Nagakawa et al [37] reported a mitral valve damage not requiring surgery. Thirty-one studies reported absence of any procedural complication. In the remaining 7 studies, the rate of procedural complications varied between $5.9 \%$ and $33 \%$. No cardiac tamponades or procedural 
deaths were reported. Overall, $0.2 \%(\mathrm{n}=2)$ of patients developed either persistent or transient complete atrio-ventricular block, $0.2 \%(\mathrm{n}=2)$ developed LBBB, $0.5 \%(\mathrm{n}=4)$ had VF arrest, $0.1 \%(n=1)$ required permanent pacemaker implant and only $0.3 \%(n=3)$ was reported to have groin complications. No complications occurred in the remaining $98.6 \%$ of patients.

\section{Discussion}

Our review confirms the high success rate for catheter ablation of FVT $(93.5 \%$ freedom of FVT after multiple procedures) with a low complication rate (observed incidence of complications was $1.4 \%$ ), with no procedural deaths or need of urgent cardiac surgery. The success rate after the index procedure was $87 \%$. As much as $9.3 \%$ of patients in this review had had a previous attempt or failed catheter ablation for FVT prior to the index procedure. Unfortunately, none of the studies included in this review reported specifically the procedural outcome in subjects with previous FVT ablation compared to naïve patients. These results support the overall efficacy and safety of catheter ablation in a FVT patient population where arrhythmia relapse had occurred in $84 \%$ of those treated with anti-arrhythmic agents. To the best of our knowledge, this is the first systematic review analysing the available literature and data on this topic.

Catheter ablation is a well-established treatment for FVT. However, evidence supporting its efficacy is limited to small non-randomised observational studies. This is likely due to the low prevalence as well as good prognosis of FVT. Medical treatment (i.e. verapamil) has been shown to be efficacious for the termination of acute episodes and prevention of recurrence in small case-series [49-52].

We have identified no studies comparing long-term medical therapy versus catheter ablation for preventing FVT relapses. However, most patients in this analysis had previously relapsed while on anti-arrhythmic drugs. According to American Heart Association/American College of Cardiology/Heart Rhythm Society guidelines, catheter ablation is useful in patients with 
verapamil-sensitive, idiopathic left VT related to interfascicular reentry for whom antiarrhythmic medications are ineffective, not tolerated, or not the patient's preference (class of recommendation I, level of evidence B-NR) [53]. Current guidelines from European Society of Cardiology recommend catheter ablation by experienced operators as a first-line treatment in symptomatic patients with idiopathic LV tachycardia (class of recommendation I, level of evidence B) [54].

Non-inducibility of FVT during electrophysiological study is often considered a pitfall of catheter ablation strategy and, in the studies included in this analysis, this represented the most common reason for deferring ablation. Earlier reports did show difficulty in induction of FVT in approximately $25-40 \%$ of the patients [14]. Our analysis suggests that FVT was inducible or incessant in $91 \%$ of cases, although occasionally VT was not sustained for long enough to enable mapping during tachycardia (often because of contact inhibition). Interestingly, ablation was performed during SR in almost half of the patients included in our analysis, usually targeting Purkinje-potentials (PP) in the interventricular septum region [19] [21, 22] [24, 25] [41-43], though delayed diastolic potentials or areas with best pace-mapping have been used as alternative targets [18] [23] [26, 27] [39] [43] [48]. A sub-analysis of our data did not demonstrate a difference in the long-term efficacy of ablation during SR vs FVT. Noninducibility or lack of sustained inducibility of FVT during the procedure therefore should not be a contraindication for performing catheter ablation.

Interestingly, studies that included paediatric-only populations did demonstrate a trend for poorer procedural results compared to those enrolling adults only. A possible explanation could be a less aggressive ablation approach likely to have been adopted in children.

Tridimensional mapping system was used in all the series published in the last 11 years. Our data suggest that availability of 3-D mapping system led to an improvement in procedural success compared to fluoroscopy-only procedures, especially after the index procedure. 


\section{Limitations}

The main limitation of this study was the inability to analyse data from case-control design due to the absence of control groups in all studies. However, given the low efficacy of medical therapy and the high success rate and safety of catheter ablation, randomised controlled trials comparing these different treatment options might not be ethically appropriate. The efficacy of antiarrhythmic therapy reported in the present study could be underestimated, given that only selected patients were included (i.e., only patients undergone to catheter ablation). Most of the studies included were retrospective, single-centre and included small cohorts. No studies provided comparative data on outcomes using different mapping and ablation strategies (e.g., mapping and ablating in FVT versus SR, or mapping earliest PP versus mechanical termination, or use of irrigated versus non-irrigated catheters). Data regarding procedure duration and fluoroscopy time are missing in several studies and therefore no comparison between ablation using irrigated versus non-irrigated catheters or 3-D mapping versus fluoroscopy only was possible.

\section{Conclusions}

Data derived from observational non-controlled case series, with low-methodological quality, suggest that catheter ablation is a safe and effective treatment for FVT, with a 93.5\% success rate after multiple procedures in patients with previously failed anti-arrhythmic therapy. Ablation during FVT represents the first-line and most commonly used approach; however, a strategy of mapping and ablation during SR displayed comparable procedural results to actively mapping patients in FVT and should therefore be considered in selected cases where FVT is not inducible. 


\section{Acknowledgements}

RJS has had research agreements and speaker fees from Abbott, Medtronic, Boston Scientific and Biosense Webster. PDL has received educational grants from Medtronic and Boston Scientific. MF has received speaker fees from Biotronik and Medtronic and owns stocks of Epicardio ltd. All other authors have reported that they have no relationships relevant to the contents of this paper to disclose.

\section{Tables}

Table 1. Baseline characteristics of studies

Table 2. Procedural characteristics

Table 3. Procedural complications and outcomes post-FVT ablation

Table 4. Sub-group Analyses

S-Table 1. Quality Assessment Scale for Case Series Studies - National Heart, Lung, and Blood Institute

S-Table 2. Supplementary procedural data

\section{Legends to figures}

Figure 1. Twelve-lead electrocardiogram of left-posterior fascicular ventricular tachycardia

Figure 2. Flowchart diagram illustrating study selection methodology

Figure 3. Forest plots assessing success rate a) after index procedure, b) after multiple procedures 


\section{References}

[1] Zipes DP, Foster PR, Troup PJ, Pedersen DH. Atrial induction of ventricular tachycardia: reentry versus triggered automaticity. Am J Cardiol. 1979;44:1-8. 
[2] Belhassen B, Rotmensch HH, Laniado S. Response of recurrent sustained ventricular tachycardia to verapamil. Br Heart J. 1981;46:679-82.

[3] Nogami A. Purkinje-related arrhythmias part I: monomorphic ventricular tachycardias. Pacing Clin Electrophysiol. 2011;34:624-50.

[4] Gaita F, Giustetto C, Leclercq JF, Haissaguerre M, Riccardi R, Libero L, et al Idiopathic verapamil-responsive left ventricular tachycardia: clinical characteristics and longterm follow-up of 33 patients. Eur Heart J. 1994;15:1252-1260.

[5] Ohe T, Aihara N, Kamakura S, Kurita T, Shimizu W, Shimomura K. Long-term outcome of verapamil-sensitive sustained left ventricular tachycardia in patients without structural heart disease. J Am Coll Cardiol. 1995;25:54.

[6] Nakagawa M, Takahashi N, Nobe S, Ichinose M, Ooie T, Yufu F, et al. Gender differences in various types of idiopathic ventricular tachycardia. $J$ Cardiovasc Electrophysiol. 2002;13:633-638.

[7] Liu Y, Fang Z, Yang B, Kojodjojo P, Chen H, Ju W, Cao K, et al. Catheter Ablation of Fascicular Ventricular Tachycardia Long-Term Clinical Outcomes and Mechanisms of Recurrence. Circ Arrhythm Electrophysiol. 2015;8:1443-51.

[8] Liu Q, Shehata M, Jiang R, Yu L, Chen S, Zhu J, et al. Macroreentrant Loop in Ventricular Tachycardia From the Left Posterior Fascicle: New Implications for Mapping and Ablation. Circ Arrhythm Electrophysiol. 2016;9(9). 
[9] Nogami A. What Is the Real Identity of the Mysterious Potential P1, and What Is the Most Important Segment of the Fascicular Ventricular Tachycardia Circuit? Circ Arrhythm Electrophysiol. 2016;9:e04517.

[10] Menzies D. Systematic reviews and meta-analyses. The international journal of tuberculosis and lung disease: the official journal of the International Union against Tuberculosis and Lung Disease 2011;15:582-593.

[11] National Heart LaBI. Quality Assessment Tool for Case Series Studies. https://www.nhlbi.nih.gov/health-pro/guidelines/in-develop/cardiovascular-riskreduction/tools/case_series

[12] Hutton B, Salanti G, Caldwell DM, Chaimani A, Schmid CH, Cameron C, et al. The PRISMA extension statement for reporting of systematic reviews incorporating network metaanalyses of health care interventions: checklist and explanations. Annals of internal medicine. 2015;162:777-784.

[13] Guo XG, Liu X, Zhou GB, Sun Q, Yang JD, Luo B, et al. Clinical, electrocardiographic, and electrophysiological characteristics of left upper septal fascicular ventricular tachycardia. Europace. 2017;doi: 10.1093/europace/euw429. [Epub ahead of print]

[14] Gopi A, Nair S, Shelke A, Saggu DK, Yalagudri S, Reddy P, Narasimhan C. A stepwise approach to the induction of idiopathic fascicular ventricular tachycardia. $J$ Interv Card Electrophysiol. 2015;44:17-22. 
[15] Collins KK, Schaffer MS, Liberman L, Saarel E, Knecht M, Tanel RE, et al. Fascicular and nonfascicular left ventricular tachycardias in the young: an international multicenter study. J Cardiovasc Electrophysiol. 2013;24:640-8.

[16] Magalhaes S, Gonçalves H, Primo J, Sá AP, Silva P, Rosas R, Gama V. Fascicular Ventricular Tachycardia: Experience with Radiofrequency Ablation. Rev Port Cardiol. 2006;25:485-97.

[17] Talib AK, Nogami A, Nishiuchi S, Kowase S, Kurosaki K, Matsui Y, et al. VerapamilSensitive Upper Septal Idiopathic Left Ventricular Tachycardia: Prevalence, Mechanism, and Electrophysiological Characteristics. JACC Clin Electrophysiol. 2015 Oct;1(5):369-380.

[18] Topilski I, Glick A, Belhassen B. Idiopathic left ventricular tachycardia with a right bundle branch block morphology and left axis deviation ("Belhassen type"): results of radiofrequency ablation in 18 patients. Isr Med Assoc J. 2004 Apr;6(4):195-200.

[19] Chen H, Zhang F, Yang B, Shehata M, Ju W, Yang G, et al. A novel method to identify the origin of ventricular tachycardia from the left fascicular system. Heart Rhythm. 2016;13:686-94.

[20] Kataria V, Yaduvanshi A, Kumar M, Nair M. Demonstration of posterior fascicle to myocardial conduction block during ablation of idiopathic left ventricular tachycardia: An electrophysiological predictor of long-term success. Heart Rhythm. 2013;10:638-45. 
[21] Shin WS, Lee MY, Jang SW, Kim JH, Yoon HJ, Jin SW, et al. The significance of repetitive ventricular responses induced by radiofrequency energy application for idiopathic left ventricular tachycardia. J Korean Med Sci. 2010 Jun;25(6):86874.

[22] Fishberger SB, Olen MM, Rollinson NL, Rossi AF. Creation of Partial Fascicular Block: An Approach to Ablation of Idiopathic Left Ventricular Tachycardia in the Pediatric Population. Pacing Clin Electrophysiol. 2015;38:209-15.

[23] Katritsis D, Heald S, Ahsan A, Anderson MH, Camm AJ, Ward DE, Rowland E. Catheter ablation for successful management of left posterior fascicular tachycardia: an approach guided by recording of fascicular potentials. Heart. 1996;75:384-388.

[24] Luo B, Zhou G, Guo X, Liu X, Yang J, Sun Q, et al. Long-term outcome of catheter ablation for left posterior fascicular ventricular tachycardia with the development of left posterior fascicular block and characteristics of repeat procedures. Int $J$ Cardiol. 2017;236:203-208.

[25] Zhan XZ, Liang YH, Xue YM, Shehata M, Liao HT, Fang XH, et al. A new electrophysiologic observation in patients with idiopathic left ventricular tachycardia. Heart Rhythm. 2016 Jul;13(7):1460-7.

$$
\text { Liu XY, Wei W, Chu JM, Wang LX, Zhao YJ, Wang J, et al. }
$$
Variations of electroanatomic substrates and markers of successful ablation in idiopathic left ventricular tachycardia: role of electroanatomic substrates and potential mechanism of tachyc ardia. J Cardiovasc Med (Hagerstown). 2014 Aug;15(8):659-67. 
[27] Chu J, Sun Y, Zhao Y, Wei W, Wang J, Liu X, et al. Identification of the Slow Conduction Zone in a Macroreentry Circuit of Verapamil-Sensitive Idiopathic Left Ventricular Tachycardia Using Electroanatomic Mapping. J Cardiovasc Electrophysiol. 2012;23:840-5.

[28] He B, Jiang H, Lu Z, Zhang M, Hu X, Yang B, et al. Feasibility and safety of transradial approach for catheter ablation of idiopathic left ventricular tachycardia. Clin Res Cardiol. 2011 Jan;100(1):37-43.

[29] Arya A, Haghjoo M, Emkanjoo Z, Fazelifar AF, Dehghani MR, Heydari A, Sadr-Ameli MA. Comparison of presystolic purkinje and late diastolic potentials for selection of ablation site in idiopathic verapamil sensitive left ventricular tachycardia. J Interv Card Electrophysiol. 2004 Oct;11(2):135-41.

[30] Ouyang F, Cappato R, Ernst S, Goya M, Volkmer $\quad$ M, Hebe J, et al. Electroanatomic substrate of idiopathic left ventricular tachycardia: unidirectional block and macroreentry within the purkinje network. Circulation. 2002 Jan 29;105(4):462-9.

[31] Aiba T, Suyama K, Aihara N, Taguchi A, Shimizu W, Kurita T, Kamakura S. The role of Purkinje and pre-Purkinje potentials in the reentrant circuit of verapamil-sensitive idiopathic LV tachycardia. Pacing Clin Electrophysiol. 2001 Mar;24(3):333-44.

[32] Miyauchi Y, Kobayashi Y, Ino T, Atarashi H. Identification of the slow conduction zone in idiopathic left ventricular tachycardia. Pacing Clin Electrophysiol. 2000;23:481-7. 
[33] Nogami A, Naito S, Tada H, Taniguchi K, Okamoto Y, Nishimura S, et al. Demonstration of diastolic and presystolic Purkinje potentials as critical potentials in a macroreentry circuit of verapamil-sensitive idiopathic left ventricular tachycardia. J Am Coll Cardiol. 2000;36:81123.

[34] Tsuchiya T, Okumura K, Honda T, Honda T, Iwasa A, Yasue H, Tabuchi T. Significance of late diastolic potential preceding Purkinje potential in verapamil-sensitive idiopathic left ventricular tachycardia. Circulation. 1999;99:2408-13.

[35] Nogami A, Naito S, Tada H, Oshima S, Taniguchi K, Aonuma K, Iesaka Y. Verapamilsensitive left anterior fascicular ventricular tachycardia: results of radiofrequency ablation in six patients. J Cardiovasc Electrophysiol. 1998;9:1269-78.

[36] Zardini M, Thakur RK, Klein GJ, Yee R. Catheter ablation of idiopathic left ventricular tachycardia. Pacing Clin Electrophysiol. 1995;18:1255-65.

[37] Nakagawa H, Beckman KJ, McClelland JH, Wang X, Arruda M, Santoro I, et al. Radiofrequency catheter ablation of idiopathic left ventricular tachycardia guided by a Purkinje potential. Circulation. 1993;88:2607-17.

[38] Ma W, Lu F, Shehata M, Wang X, Zhang F, Chen B, et al. Catheter Ablation of Idiopathic Left Posterior Fascicular Ventricular Tachycardia: Predicting the Site of Origin via Mapping and Electrocardiography. Circ Arrhythm Electrophysiol. 2017;10(11); pii: e005240. 
[39] Letsas KP, Efremidis M, Vlachos K, Asvestas D, Georgopoulos S, Xydonas S, et al. Catheter ablation of idiopathic fascicular ventricular tachycardia: The role of isolated diastolic potentials during mapping in sinus rhythm. Int J Cardiol. 2015;201:212-4.

[40] Park J, Kim YH, Hwang C, Pak HN. Electroanatomical Characteristics of Idiopathic Left Ventricular Tachycardia and Optimal Ablation Target during Sinus Rhythm: Significance of Preferential Conduction through Purkinje Fibers. Yonsei Med J. 2012 Mar 1; 53(2): 279-288.

[41] Wissner E, Menon SY, Metzner A, Schoonderwoerd B, Nuyens D, Makimoto H, et al. Long-Term Outcome After Catheter Ablation for Left Posterior Fascicular Ventricular Tachycardia Without Development of Left Posterior Fascicular Block. J Cardiovasc Electrophysiol. 2012;23:1179-84.

[42] Ma FS, Ma J, Tang K, Han H, Jia YH, Fang PH, et al. Left posterior fascicular block: a new endpoint of ablation for verapamil-sensitive idiopathic ventricular tachycardia. Chin Med $J$ (Engl). 2006;5;119:367-72.

[43] Lin D, Hsia HH, Gerstenfeld EP, Dixit S, Callans DJ, Nayak H, et al. Idiopathic fascicular left ventricular tachycardia: Linear ablation lesion strategy for noninducible or nonsustained tachycardia. Heart Rhythm. 2005;2:934-9.

[44] Gupta AK, Kumar AV, Lokhandwala YY, Vora AM, Maheshwari A, Thakur RK. Primary Radiofrequency Ablation for Incessant Idiopathic Ventricular Tachycardia. Pacing Clin Electrophysiol. 2002;25:1555-60. 
[45] Wen MS, Yeh SJ, Wang CC, Lin FC, Wu D. Successful radiofrequency ablation of idiopathic left ventricular tachycardia at a site away from the tachycardia exit. J Am Coll Cardiol. 1997;30:1024-31.

[46] Wen MS, Yeh SJ, Wang CC, Lin FC, Chen IC, Wu D. Radiofrequency ablation therapy in idiopathic left ventricular tachycardia with no obvious structural heart disease. Circulation. 1994;89:1690-6.

[47] Bennet DH. Experience with radiofrequency catheter ablation of fascicular tachycardia. Heart. 1997;77:104-107.

[48] Suzuki T, Nakamura Y, Yoshida S, Yoshida Y, Nakamura K, Sasaki T, Fujino M, Kawasaki Y, Ehara E, Murakami Y, Shintaku H. Radiofrequency catheter ablation of idiopathic left anterior fascicular ventricular tachycardia in children. Heart Rhythm. $2014 ; 11: 1948-56$.

[49] Ohe T, Shimomura K, Aihara N, Kamakura S, Matsuhisa M, Sato I, Nakagawa H, Shimizu A. Idiopathic sustained left ventricular tachycardia: clinical and electrophysiologic characteristics. Circulation. 1988;77:560.

[50] German LD, Packer DL, Bardy GH, Gallagher JJ. Ventricular tachycardia induced by atrial stimulation in patients without symptomatic cardiac disease. Am $J$ Cardiol. $1983 ; 52: 1202$. 
[51] Klein GJ, Millman PJ, Yee R. Recurrent ventricular tachycardia responsive to verapamil. Pacing Clin Electrophysiol. 1984;7:938.

[52] Ward DE, Nathan AW, Camm AJ. Fascicular tachycardia sensitive to calcium antagonists. Eur Heart J. 1984;5:896.

[53] Al-Khatib SM, Stevenson WG, Ackerman MJ, et al. 2017 AHA/ACC/HRS Guideline for Management of Patients With Ventricular Arrhythmias and the Prevention of Sudden Cardiac Death: Executive Summary: A Report of the American College of Cardiology/American Heart Association Task Force on Clinical Practice Guidelines and the Heart Rhythm Society. Circulation. 2017; pii: CIR.0000000000000548. doi: 10.1161/CIR.0000000000000548. [Epub ahead of print]

[54] Priori SG, Blomström-Lundqvist C, Mazzanti A, et al. 2015 ESC Guidelines for the management of patients with ventricular arrhythmias and the prevention of sudden cardiac death: The Task Force for the Management of Patients with Ventricular Arrhythmias and the Prevention of Sudden Cardiac Death of the European Society of Cardiology (ESC). Endorsed by: Association for European Paediatric and Congenital Cardiology (AEPC). Eur Heart J. 2015;36:2793-867. 